\title{
La modelación matemática: Un ejemplo en el contexto cafetero ${ }^{1}$
}

\author{
Mathematical modeling: An example in the context coffee
}

A modelação matemática: Um exemplo no contexto de café

Recibido: mayo de 2013

Aprobado: agosto de 2013
John Fredy Sánchez Betancur ${ }^{2}$

Jorge Didier Obando Montoya ${ }^{3}$

Lina María Muñoz Mesa ${ }^{4}$

Jhony Alexander Villa-Ochoa ${ }^{5}$

\begin{abstract}
Resumen
En este documento analizamos un episodio que surge de una investigación en el marco de la Maestría en Educación (Matemática) de la Universidad de Antioquia. En la experiencia participaron cinco estudiantes de grado décimo de una institución educativa que se encuentra ubicada en un municipio reconocido por su producción cafetera; fue a partir de esta realidad, que implementamos un proceso de la modelación matemática en el contexto del cultivo del café. En este artículo presentamos un episodio que se extrae de la fase en la cual los estudiantes fueron invitados a elegir, acorde con sus intereses, un fenómeno o situación en dicho contexto para ser modelado matemáticamente. El episodio refleja que a pesar que los estudiantes se comprometen con la toma de decisiones sobre la temática y lograron profundizar en su conocimiento del contexto, aún siguen reconociendo como aporte principal de la modelación matemática una visión de las matemáticas como un área de presencia y utilidad en muchas de las situaciones sociales y culturales.
\end{abstract}

Palabras clave: Educación Matemática y otras disciplinas; contexto cafetero; variables; aprendizaje; procesos cognitivos; modelación y modelo.

\begin{abstract}
\end{abstract}
In this paper we analyze an episode that grew out of research in the framework of the Master of Education (Mathematics) from the University of Antioquia. In the experience involving five tenth grade students of an educational institution which is located in a city known for its coffee production, it was from this reality, we implemented a process of mathematical modeling in the context of growing coffee. In this paper we present

\footnotetext{
1 Artículo de Investigación

2 Universidad de Antioquia. Medellin, Colombia. Contacto: jofresanbeta@hotmail.com

3 Universidad de Antioquia. Medellin, Colombia. Contacto: jdom.6@hotmail.com

4 Universidad de Antioquia. Medellin, Colombia. Contacto: limamu07@gmail.com

5 Universidad de Antioquia. Medellin, Colombia. Contacto: javo@une.net.co
} 
an episode that is extracted from the phase in which students were invited to choose, according to their interests, a phenomenon or situation in this context to be modeled mathematically. The episode reflects that although students are committed to making decisions on the issue and managed to deepen their knowledge of the context, even still recognized as the main contribution of mathematical modeling a vision of mathematics as a presence and utility area in many of the social and cultural situations.

Keywords: Mathematics Education and other disciplines; coffee context, variables, learning cognitive processes modeling and model.

\section{Resumo}

Neste artigo analisamos um episódio que cresceu a partir de pesquisas no âmbito do Mestrado em Educação (Matemática) da Universidade de Antioquia. Na experiência envolvendo cinco alunos do décimo de uma instituição de ensino que está localizado em uma cidade conhecida por sua produção de café, foi a partir dessa realidade, nós implementamos um processo de modelagem matemática no contexto da cultura do café. Neste artigo apresentamos um episódio que é extraído a partir da fase em que os alunos foram convidados a escolher, de acordo com os seus interesses, um fenômeno ou situação, neste contexto, ser modelado matematicamente. $\mathrm{O}$ episódio reflete que, apesar de os estudantes estão empenhados em tomar decisões sobre o assunto e conseguiu aprofundar o conhecimento do contexto, mesmo ainda reconhecida como a principal contribuição da modelagem matemática de uma visão da matemática como uma presença e área de serviço em muitas das situações sociais e culturais.

Palavras-chave: Educação Matemática e outras disciplinas; contexto café, as variáveis, os processos de aprendizagem de modelagem cognitiva e modelo.

\section{El estudio. Consideraciones, propósito y camino metodológico}

En Colombia el Ministerio de Educación Nacional a través de sus diferentes publicaciones para el área de matemáticas (Colombia, 1998, 2006) ha llamado la atención sobre la necesidad que los procesos desarrollados en el aula de clase se constituyan en espacios en donde la interacción entre docentes y estudiantes permita construir y validar conocimiento, ejercer iniciativa desde la crítica y la aplicación de ese conocimiento en diversas situaciones y contextos. Si bien desde Colombia (1998) se viene proponiendo el uso de contextos matemáticos, cotidianos y de otras ciencias para el desarrollo de la actividad matemática escolar, también es cierto que, al menos para los dos últimos mencionados, no existen orientaciones explícitas para usarlos en el aula de clase.

Según Villa-Ochoa (2013) la modelación matemática y los contextos de los cuales emerge, tienen diferentes intenciones y juegan diversos roles al interior del aula de clase. En este documento asumimos aquellos en los cuales la modelación va más allá de una visión utilitarista del contexto y no se agota en la actividad cognitiva de trasladar o traducir un tópico o cuestión de un dominio extra matemático a un dominio matemático. De ese modo, a esta actividad le asignamos la responsabilidad no solo de la producción de significados matemáticos situados en un contexto extramatemático, sino también de valorar otros aspectos propios de los contextos. Con estas ideas de fondo, hemos venido desarrollando una investigación en la que nos preocupamos por 
los conocimientos que producen los estudiantes, tanto del contexto como los algebraicos, cuando se enfrentan a procesos de modelación en un contexto del cultivo cafetero.

La investigación en estos términos describe un marco metodológico centrado en las producciones de un conjunto de cinco estudiantes de grado décimo de la Institución Educativa San Juan de los Andes, cuyas familias centran principalmente su actividad económica en el sector caficultor; por esta razón, los estudiantes se motivaron a desarrollar sus actividades de modelación articulados a este contexto. La información en la que se fundamenta nuestra investigación se produjo a partir de observación directa, diarios de campo, diálogos, entrevistas con los estudiantes, así como en los documentos producidos por ellos mismos. En una de las fases de la investigación, y sin perder de vista el interés de los estudiantes por el cultivo de café, los invitamos a elegir la situación o fenómeno en la que quisieran indagar a través de la modelación matemática. Uno de los episodios producidos por los estudiantes se analiza en este documento.

\section{Una breve descripción de algunos referentes teóricos}

Coherente con los anteriores planteamientos, observamos en la modelación matemática un proceso de estudio de situaciones (en el contexto cafetero) y desde el cual pueden producirse modelos matemáticos y otros conocimientos propios del contexto de la situación. Para observar dicho proceso, vemos en Villa-Ochoa (2007) y Biembengut \& Hein (2004) la posibilidad de algunas orientaciones generales para desarrollar la modelación matemática; así mismo, rescatamos la experiencia de Berrío (2012) para resaltar el papel del conocimiento que emerge en la modelación matemática. En contraste con los autores anteriores, encontramos tanto en Araújo (2009) como en Rosa, Reis \& Orey (2012) aspectos que han puesto de relieve el papel de la modelación matemática no solo para interpretar situaciones que emergen de la realidad, sino también como una manera de proporcionar a los estudiantes formas de actuación que les permitan posicionarse de manera crítica frente a las demandas sociales, modificar y transformar el mundo, así como servir de apoyo tecnológico para cuestionar la matemática misma y su rol en la sociedad.

\section{El Episodio}

Este episodio se desprende de una experiencia en la cual los estudiantes se preocuparon por observar las temáticas o situaciones a indagar a través de la modelación. Tópicos como la fertilización, recolección, secado y las plagas emergieron en las discusiones. En particular, para este documento presentaremos el análisis realizado por uno de los participantes frente al fenómeno de las plagas, en especial la broca.

Para proceder al estudio del fenómeno, se les invitó a consultar información sobre el ciclo biológico de la broca. La información consultada se presenta en el siguiente fragmento:

En Colombia se han realizado varios estudios sobre el ciclo biológico de la broca (...). Aunque los datos obtenidos presentan algunas diferencias, esto se debe a las condiciones ambientales donde se han realizado, en especial, la temperatura. La hembra después de salir de la pupa está lista para aparearse y la oviposición la inicia tres días más tarde. Cada hembra pone entre 2 y 3 huevos por día por un periodo de 15 días, es decir un promedio de 43 huevos por postura. El ciclo total de huevo (incubación, larva, pre-pupa y pupa) a adulto en Colombia se estima en 27,5 días; sin embargo, el tiempo generacional, o sea el tiempo que tarda en iniciarse otra generación del insecto, se estima en 45 días a $22^{\circ} \mathrm{C}$, por lo tanto, en aquellos cultivos cuya producción es todo el año, la broca puede superar las 8 generaciones por año. La relación de sexos es de un macho por cada 10 hembras. En cada grano la supervivencia de la descendencia está constituida por 25-30 individuos en todos los estados de desarrollo. (Bustillo Pardey, 2006).

La experiencia en el aula mostró que los estudiantes poseen conocimientos sobre el fenómeno los cuales son derivados de su contacto con el mismo; sin 
Ilustración 1. Proceso realizado por el estudiante con seudónimo Antonio.
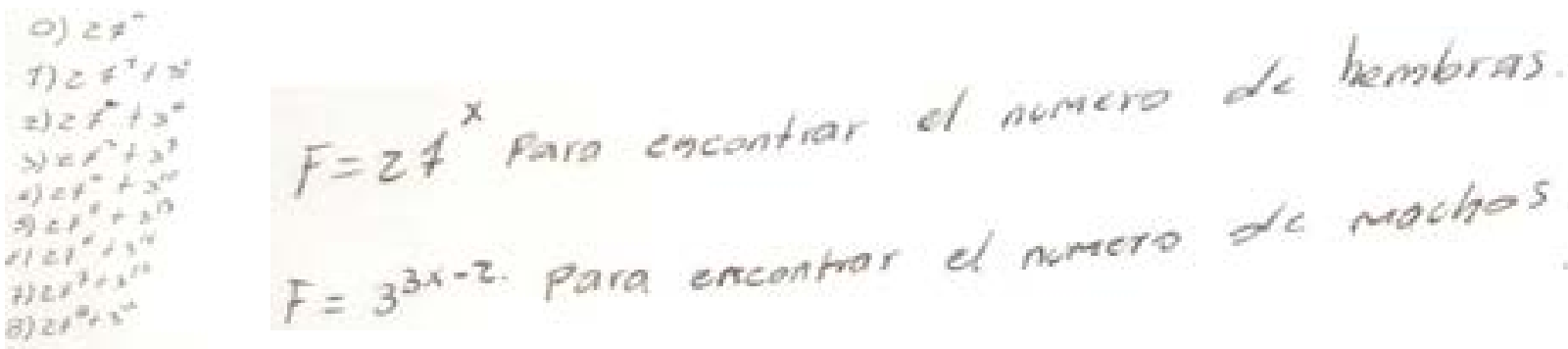

Fuente: Elaboración propia

embargo, no son suficientes para atender a la complejidad del mismo. Por esta razón, en discusión con los estudiantes, se opta asumir unos datos simplificados y así comprometer al grupo en su análisis.
Del texto los estudiantes producen dos modelos diferentes (ver ilustración 1), uno sobre las brocas hembras que nacen en cada generación y, el otro, sobre las brocas machos que nacen en cada generación.

\begin{tabular}{|c|c|}
\hline $\begin{array}{l}\text { "Aprendi con este trabajo que una persona Debe } \\
\text { poner más interés por la matemática porque a } \\
\text { través de la matemática se puede llegar a explicar } \\
\text { muchas cosas"..."veo que la matemática es una } \\
\text { ciencia que se puede aplicar en muchas cosas" }\end{array}$ & $\begin{array}{l}\text { "Estos modelos pueden permitir crear en las } \\
\text { personas un mayor conocimiento sobre las plagas } \\
\text { y la fertilización de los cultwos principalmente" }\end{array}$ \\
\hline $\begin{array}{l}\text { La redacción y ortografia se encuentran fieles a la } \\
\text { producción original de Carlos }\end{array}$ & $\begin{array}{l}\text { La redacción y ortografia se encuentran fieles a la } \\
\text { producción de Emesto }\end{array}$ \\
\hline
\end{tabular}

Tabla 1. Comentarios efectuados por Carlos y Eraesto

Estos modelos muestran unas relaciones que surgen del fenómeno analizado y una representación matemática, descritos desde procedimientos aritméticos y expresiones que generalizan estos comportamientos numéricos.

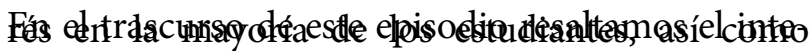
otras dinámicas en el aula de clase en las cuales se comparten ideas, exponen y argumentan puntos de vista, además se evidenció que tenían algunos conocimientos sobre la problemática abordada, y la necesidad de seguir ampliándola para comprender de una manera más profunda cada fenómeno. En la siguiente tabla trascribimos algunos de los comentarios extraídos de los documentos.

En el desarrollo de la modelación matemática se resalta la necesidad de retomar algunos conocimientos que los estudiantes debían de haber estudiado en grados anteriores; así mismo, se observa la valoración que ellos hacen frente a su participación en las decisiones sobre el trabajo matemático escolar. A pesar de ello, limitaciones en términos del tiempo para el desarrollo de la modelación, los conocimientos matemáticos previos, y otras de las reportadas por Biembengut \& Hein (2004) también se hicieron evidentes.

\section{Reflexión final}

La participación de los estudiantes en las decisiones acerca de las situaciones y fenómenos a estudiar en el aula de clase parece ser un aspecto que, además de una motivación inicial para la actividad matemática, también es una manera en la que ellos se asumen como agentes claves en el desarrollo de esta área en la escuela. A pesar de este aspecto y de la posibilidad de comprender mejor los contextos en el que los estudiantes se desenvuelven, el primer y principal reconocimiento que ellos hicieron en la modelación fue a la posibilidad de ver la matemática como 
un área aplicada o con posibilidades de "explicar" muchas cosas. Este tipo de aspectos pueden corresponder bien sea porque la metodología con la que se desarrolló la experiencia de modelación en este episodio les haya permitido a los estudiantes observar una ruptura de las rutinas en las que clásicamente se envuelve las matemáticas escolares, o porque se hayan encuadrado con ciertos aspectos de la ideología de la certeza (Borba \& Skovsmose, 1997) en la cual la matemática ha de ser reconocida por su capacidad de incurrir en muchas de las facetas sociales y humanas. Sea cual sea el caso, este trabajo sugiere que para lograr ver la modelación mas allá de una aplicación matemática, es necesario mayores discusiones en el aula de clase en la cual los estudiantes puedan observar otros roles de las matemáticas en la sociedad y sobre todo, re-valoren su propio rol como "agentes productores" de matemática escolar.

\section{Referencias}

Araújo, J. L. (2009). Uma abordagem Sócio-Crítica da modelagem matemática: a perspectiva da educação matemática crítica. ALEXANDRIA Revista de Educação em Ciência e Tecnologia, 2(2), 55-68.

Berrío, M. (2012). Elementos que intervienen en la construcción que hacen los estudiantes frente a los modelos matemáticos. El caso del cultivo de café. (Maestría en Enseñanza de las Ciencias Exactas y Naturales Tesis de maestría no publicada), Universidad Nacional de Colombia, Medellín.

Biembengut, M., \& Hein, N. (2004). Modelación matemática y los desafíos para enseñar matemática. Educación Matemática, 16(2), 105-125.

Borba, M. C., \& Skovsmose, O. (1997). The ideology of certainty in mathematics education. For the learning of mathematics, 17(3), 17- 23.

Bustillo Pardey, A. E. (2006). Una revisión sobre la broca del café, Hypothenemus hampei (Coleoptera: Curculionidae: Scolytinae), en Colombia. Revista Colombiana de Entomología, 32(2), 101-116.

Colombia, M. d. E. N. (1998). Lineamientos Curriculares para el área de Matemáticas. Santa fe de Bogotá: Cooperativa Editorial Magisterio.

Colombia, M. d. E. N. (2006). Estándares Básicos de Competencia. Bogotá: Cooperativa Editorial Magisterio.

Rosa, M., Reis, F., \& Orey, D. (2012). A Modelagem Matemática Crítica nos Cursos de Formação de Professores de Matemática Acta Scientiae, 14(2), 159-184.

Villa-Ochoa, J. A. (2007). La Modelación como Proceso en el Aula de Matemáticas: Un Marco de Referencia y un Ejemplo. Tecno Lógicas, 0(19), 63-86.

Villa-Ochoa, J. A. (2013). Miradas y actuaciones sobre la modelación matemática en el aula de clase. Paper presented at the VIII Conferência Nacional sobre Modelagem Matemática na Educação Matemática Santa Maria-RS, Brasil. 